\title{
A NOTE ON THE RANK OF THE 2-CLASS GROUP OF THE HILBERT 2-CLASS FIELD OF SOME REAL QUADRATIC NUMBER FIELDS
}

\author{
ELLIOT BENJAMIN \\ Department of Psychology \\ Capella University \\ USA \\ e-mail ben496@prexar.com
}

\begin{abstract}
Let $k$ be a real quadratic number field with 2-class group $C_{2}(k)$ isomorphic to $Z / 2^{m} Z \times Z / 2^{n} Z, m \geq 2, n \geq 2$, such that the discriminant of $k$ is divisible by only positive prime discriminants. Let $k^{1}$ be the Hilbert 2-class field of $k$, and $k_{1}, k_{2}, k_{3}$ be the three unramified quadratic extensions of $k$. We prove that if the 2-class number of $k$ is equal to the 2-class number of $k_{i}$ for $i=1,2$, and 3, then either $\left|C_{2}\left(k^{1}\right)\right|=2$ or $\operatorname{rank}\left(C_{2}\left(k^{1}\right)\right) \geq 3$.
\end{abstract}

\section{Introduction}

Let $k$ be an algebraic number field, $C_{2}(k)$ denote the 2-Sylow subgroup of its ideal class group $C(k)$, and $k^{1}$ denote the Hilbert 2-class field of $k$ (in the wide sense). Let $k^{n}$ (for a nonnegative integer $n$ ) be 2010 Mathematics Subject Classification: 11R29, $20 \mathrm{D} 15$.

Keywords and phrases: real quadratic number field, Hilbert 2-class field, discriminant, 2-rank, unramified quadratic extension, commutator subgroup, noncyclic class group.

Received March 31, 2018; Revised April 28, 2018

(C) 2018 Scientific Advances Publishers 
defined inductively as $k^{0}=k$ and $k^{n+1}=\left(k^{n}\right)^{1}$. Denoting by $C$ the containment symbol, we define $k^{0} C k^{1} C k^{2} C \ldots k^{n} C \ldots$ to be the 2-class field tower of $k$. We say that the tower is finite if $k^{n}=k^{n+1}$ for some $n$, with length $n$ if $n$ is minimal, and infinite otherwise.

Currently there is no known decision procedure to determine whether or not the 2-class field tower (or the $p$-class field tower for any prime $p$ ) of an algebraic number field $k$ is infinite. It is known though, by purely group theoretical means (cf. [6], [12]) that the 2-class field tower is finite of length at most 3 when the rank, i.e., the minimal number of generators, of $C_{2}\left(k^{1}\right)$ is less than or equal to 2 ; and that if we also have $\operatorname{rank}\left(C_{2}(k)\right)=2$ (resp., 1$)$, then the tower is finite of length at most 2 (resp., 1).

We concentrate here on the case of real quadratic number fields $k$ such that its discriminant $d_{k}$ is divisible by only positive prime discriminants. In [5], we characterized all real quadratic number fields $k$ for which $C_{2}\left(k^{1}\right)$ is trivial (hence of rank 0). In [1, 2, 4] and [8], the authors determined in particular those real quadratic number fields $k$ with $\operatorname{rank}\left(C_{2}(k)\right)=2$ such that $d_{k}$ is divisible by only positive prime discriminants, for which $C_{2}\left(k^{1}\right)$ is cyclic and nontrivial (rank 1). Using the notation $\left(2^{m}, 2^{n}\right)$ to denote $Z / 2^{m} Z \times Z / 2^{n} Z, m \geq 1, n \geq 1$, it is well known that $C_{2}\left(k^{1}\right)$ is cyclic when $C_{2}(k) \approx(2,2)$ for any number field $k$, and in [3] we were able to give complete criteria to distinguish between $\operatorname{rank}\left(C_{2}\left(k^{1}\right)\right)=2$ and $\operatorname{rank}\left(C_{2}\left(k^{1}\right)\right) \geq 3$ for a real quadratic number field $k$ when $C_{2}(k) \approx\left(2,2^{n}\right), n \geq 2$, and $d_{k}$ is divisible by only positive prime discriminants. Furthermore, in [2], we found criteria to determine when $\operatorname{rank}\left(C_{2}\left(k^{1}\right)\right)=2$ and $\operatorname{rank}\left(C_{2}\left(k^{1}\right)\right) \geq 3$ for $C_{2}(k) \approx\left(2^{m}, 2^{n}\right)$, $m \geq 2, n \geq 2$, in particular cases. Denoting $h_{2}(k)$ (resp., $h(k)$ ) to 
represent the 2-class number (resp., class number) of a field $k$, we determined for these fields $k$ that if $h_{2}\left(k_{i}\right)>h_{2}(k)$ for $i=1,2,3$, where the $k_{i}$ are the three unramified quadratic extensions of $k$, then rank $\left(C_{2}\left(k^{1}\right)\right) \geq 3$. We also formulated criteria to distinguish between rank $\left(C_{2}\left(k^{1}\right)\right)=2$ and rank $\left(C_{2}\left(k^{1}\right)\right) \geq 3$ when there exists exactly one unramified quadratic extension $k_{i}$ such that $h_{2}\left(k_{i}\right)=h_{2}(k)$ (cf. Lemma 2).

In this current note, we examine the case in which for all three unramified quadratic extensions, $k_{1}, k_{2}$, and $k_{3}$, we have $h_{2}\left(k_{1}\right)=h_{2}\left(k_{2}\right)$ $=h_{2}\left(k_{3}\right)=h_{2}(k)$. We show in this case that either $\left|C_{2}\left(k^{1}\right)\right|=2$ or rank $\left(C_{2}\left(k^{1}\right)\right) \geq 3$, and therefore we see that it may possible in this case that $k$ has infinite 2-class field tower for some fields $k$.

We obtain our main result using properties of metabelian 2-groups of rank 2 and then applying these properties to number fields, as we have done in our previous work (cf. [1, 2, 3, 4]). We start with a brief review of the group theory that we need to utilize.

\section{Properties of Some Metabelian 2-Groups of Rank 2 and Preliminary Results}

Let $G=\operatorname{Gal}\left(k^{2} / k\right)$ and $G^{\prime}$ denote the commutator subgroup of $G$. By class field theory it is well known that $G / G^{\prime} \approx \operatorname{Gal}\left(k^{1} / k\right) \approx C_{2}(k)$, and $G^{\prime} \approx \operatorname{Gal}\left(k^{2} / k^{1}\right) \approx C_{2}\left(k^{1}\right)$. We recall that a group $G$ is said to be metabelian if its commutator group $G^{\prime}$ is abelian. From our earlier work we begin with the following lemma (cf. Proposition 1 in [4]).

Lemma 1. Let $G$ be a finite metabelian 2-group such that $G / G^{\prime} \approx$ $\left(2^{m}, 2^{n}\right), m \geq 1, n \geq 1$, with two generators $a$ and $b$ such that $a^{\wedge}\left(2^{m}\right) \equiv$ $b^{\wedge}\left(2^{n}\right) \equiv 1 \bmod G^{\prime}$ (where the symbol $\wedge$ denotes exponentiation). Let 
$H_{1}=<a, b^{2}, G^{\prime}>, H_{2}=<a b, b^{2}, G^{\prime}>$, and $H_{3}=<a^{2}, b, G^{\prime}>$ be the three maximal subgroups of $G$, and $J_{0}=<a^{2}, b^{2}, G^{\prime}>=H_{1} \cap H_{2} \cap H_{3}$, which is a normal subgroup of $G$ of index 4 . Then

(i) $\left|G^{\prime}\right|=1$ if and only if $\left(G^{\prime}: H_{j}^{\prime}\right)=1$ for some (equivalently all) $j \in\{1,2,3\}$

(ii) $\left|G^{\prime}\right|=2$ if and only if $\left(G^{\prime}: J_{0}{ }^{\prime}\right)=2$.

Lemma 1 has the following immediate field theoretical corollary, where $h_{2}(k)$ (resp., $h(k)$ ) denotes the 2-class number (resp., class number) of an algebraic number field $k$ (cf. Theorem 1 in [4]).

Corollary 1. Let $k$ be a number field such that $C_{2}(k) \approx\left(2^{m}, 2^{n}\right)$, $m \geq 1, n \geq 1$. Then

(i) $h_{2}\left(k^{1}\right)=1$ if and only if $h_{2}\left(k_{j}\right)=(1 / 2) h_{2}(k)$ for any (or equivalently all) of the three unramified quadratic extensions $k_{j}$ of $k, j=1,2,3$;

(ii) $h_{2}\left(k^{1}\right)=2$ if and only $h_{2}\left(k_{0}\right)=(1 / 2) h_{2}(k)$, where $K_{0}=k_{1} k_{2} k_{3}$.

In our earlier work (cf. Theorem 1 in [2]), we made further use of group theory to obtain more information about fields $k$ as above when $C_{2}\left(k^{1}\right)$ is cyclic with $h_{2}\left(k^{1}\right) \geq 4$, and we will also make use of this group theory to obtain our current results. We let $G$ be a metabelian 2-group such that $G / G^{\prime} \approx\left(2^{m}, 2^{n}\right), m \geq 2, n \geq 2$, where $G=<a, b>: a^{\wedge}\left(2^{m}\right)$ $\equiv b^{\wedge}\left(2^{n}\right) \equiv 1 \bmod G^{\prime}$. In our previous work (cf. [2]; see also [7]), we calculated the commutator subgroups of the three maximal subgroups, $H_{1}, H_{2}, H_{3}$, and seven normal subgroups of index four, $J_{0}, J_{11}, J_{12}$, $J_{21}, J_{22}, J_{31}, J_{32}$, in $G$. We described these subgroups as follows: $H_{1}=$ $<a, b^{2}, G^{\prime}>, H_{2}=<a b, b^{2}, G^{\prime}>, H_{3}=<a^{2}, b, G^{\prime}>, J_{0}=<a^{2}, b^{2}, G^{\prime}>$, 
$J_{11}=<a, b^{4}, G^{\prime}>, J_{12}=<a b^{2}, b^{4}, G^{\prime}>, J_{21}=<a b, b^{4}, G^{\prime}>, J_{22}=<a^{3} b$, $b^{4}, G^{\prime}>, J_{31}=<a^{4}, b, G^{\prime}>, J_{32}=<a^{4}, a^{2} b, G^{\prime}>$. In order to calculate all the above commutator subgroups, we made use of the lower central series $\left\{G_{k}\right\}$ of $G$, defined inductively as $G_{1}=G, G_{2}=G^{\prime}=\left[G, G_{1}\right]$, $G_{k+1}=\left[G, G_{k}\right]$, for all $k \geq 1$, where $[A, B]=<\left\{[a, b]=a^{-1} b^{-1} a b\right\} \mid a \in A$, $b \in B>$. It is well known that the lower central series terminates in finitely many steps at the identity subgroup I for all finite $p$-groups (i.e., groups of $p$-power order for any prime $p$ are nilpotent) (cf. [10]). Furthermore, we defined $\left[x_{1}, \ldots, x_{k}\right]$ inductively on $k$ as $\left[\left[x_{1}, \ldots, x_{k-1}\right]\right.$, $x_{k}$ ] for all $x_{j} \in G$ and $k>2$.

We utilized commutator and lower central series relations to calculate the above commutator subgroups. In particular, we utilized the notation $y_{1,1}=y_{11}=[a, b]$, and for $r, s \in N$ (the natural numbers), $y_{r, s}=y_{r s}=\left[a, b, x_{1}, \ldots, x_{k}\right]$ with $x_{j}=a$ or $b, k=r+s-2$, and where $r$ (resp., $s$ ) is the number of occurrences of $a$ (resp., $b$ ) in the commutator (cf. [2]). From the commutator identities $[x y, z]=[x, z][x, z, y][y, z]$ and $[x, y z]=[x, z][x, y][x, y, z]$, we know that $y_{r s}$ is independent of the order of the $x_{1}, \ldots, x_{k} ; G_{n}=<\left\{y_{r s}: r, s \in N, r+s=n\right\}, G_{n+1}>; G_{k}=1$ for sufficiently large $k$ (see above); and thus we have $G_{2}=<\left\{y_{r s}: r, s \in N, r+s \leq k\right\}>$ (cf. [2]). We also know that the group exponent $\exp \left(G_{3} / G_{4}\right)$ divides $\exp \left(G_{2} / G_{3}\right)$, and more generally, $\exp \left(G_{n} / G_{n+1}\right)$ divides $\exp \left(G_{n-1} / G_{n}\right)$ for any $n>2$ (cf. [11], p. 266).

We utilized the above group theory to obtain a listing of all the above commutator subgroups, which enabled us to prove that if $k$ is a real quadratic number field with discriminant $d_{k}=d_{1} d_{2} d_{3}$, where the $d_{j}, j=1,2,3$, are positive prime discriminants, $C_{2}(k) \approx\left(2^{m}, 2^{n}\right), m \geq 2, n \geq 2$, and $h_{2}\left(k_{j}\right)=h_{2}(k)$ for all three unramified quadratic extensions $k_{j}$ of $k$, 
(which implies that $C_{2}\left(k^{1}\right)$ is not trivial by Corollary 1) then $C_{2}\left(k^{1}\right)$ is not cyclic with $h_{2}(k) \geq 4$ (cf. Theorem 5 in [2]). We also proved that if $k$ is as above but with $(w \log ) h_{2}\left(k_{i}\right)=h_{2}\left(k_{j}\right)=h_{2}(k), h_{2}\left(k_{1}\right)>h_{2}(k)$, then $C_{2}\left(k^{1}\right)$ is not cyclic (cf. Theorem 6 in [2]). Furthermore, we established a corollary to our group theoretical results (cf. Theorems 1, 2, 3, and Corollary 2 in [2]) to obtain the following result for the other possible two cases for the 2-class numbers of the three unramified quadratic extensions of $k$, assuming that $C_{2}\left(k^{1}\right)$ is nontrivial by means of Corollary 1 .

Lemma 2. Let $k$ be a number field such that $C_{2}(k) \approx\left(2^{m}, 2^{n}\right), m \geq 1$, $n \geq 1$, and assume that $h_{2}\left(k_{i}\right)=h_{2}(k)$ for exactly one of the three unramified quadratic extensions $k_{i}$ of $k$. Then rank $\left(C_{2}\left(\mathrm{k}^{1}\right)\right)=2$ if $h_{2}\left(k_{i j}\right)=h_{2}(k)$ and rank $\left(C_{2}\left(k^{1}\right)\right) \geq 3$ if $h_{2}\left(k_{i j}\right)>h_{2}(k)$, for either (both) unramified quadratic cyclic extensions $K_{i j}$ of $k$ containing $k_{i}$. Furthermore, if $h_{2}\left(k_{i}\right)>h_{2}(k)$ for all three $k_{i}$, then rank $\left(C_{2}\left(k^{1}\right)\right) \geq 3$.

\section{Distinguishing Between Rank $\left(C_{2}\left(k^{1}\right)\right)=2$ and $\operatorname{Rank}\left(C_{2}\left(k^{1}\right)\right) \geq 3$}

As we see from the above discussion and Lemma 2, for $k$ a real quadratic number field with $C_{2}(k) \approx\left(2^{m}, 2^{n}\right), m \geq 2, n \geq 2$, where the discriminant $d_{k}$ is divisible by only positive prime discriminants, the open questions in regard to the rank of $C_{2}\left(k^{1}\right)$ are for the cases when $h_{2}\left(k_{i}\right)=h_{2}(k)$ for all three unramified quadratic extensions $k_{i}$ of $k$, and when $h_{2}\left(k_{i}\right)=h_{2}(k)$ for exactly two of the $k_{i}$. We now establish our main result, which is that either $\left|C_{2}\left(k^{1}\right)\right|=2$ or rank $\left(C_{2}\left(k^{1}\right)\right) \geq 3$ when $h_{2}\left(k_{i}\right)=h_{2}(k)$ for all three unramified quadratic extensions $k_{i}$ of $k$ as 
above. From Lemma 3 of [2] and the proof of Theorem 2 in [2], we know that for $k$ as above with $h_{2}\left(k_{i}\right)=h_{2}(k)$ for $i=1,2,3$, we have $\left(G_{2}: G_{3}\right)=2$, and that if $\operatorname{rank}\left(G_{2}\right)=2$ then $G_{2} / G_{4} \approx(2,2)$. To establish our main result, we begin with the following lemma, whose proof is due to Chip Snyder, where for $G^{\prime}$ nontrivial, $c=\mid\{j \in\{1,2,3\}$ : $\left.\left(G^{\prime}: H_{j}^{\prime}\right)=2\right\} \mid$, and where the symbol $d(G)$ denotes the rank of a group $G$.

Lemma 3. Let $G$ be a finite metabelian 2-group of rank 2, and assume that $d\left(G_{2}\right)=2$ and $c=3$. Then $J_{0}^{\prime}=G_{4}$.

Proof. Since $\left(G_{2}: G_{3}\right)=2$ and $G_{2} / G_{4} \approx(2,2), w \log G_{3}=<y_{12}, G_{4}>$. By the presentation of $J_{0}$ we have $J_{0}^{\prime}=<y_{11}{ }^{4} y_{12}{ }^{2} y_{21}{ }^{2} y_{22},\left\{y_{r+1, s}{ }^{2}\right.$ $\left.y_{r+2, s}\right\},\left\{y_{r, s+1}^{2} y_{r, s+2}\right\}>C G_{4}$ (cf. [2]). If $G_{4}=1$, then our result is trivially true, so we assume that $G_{4} \neq 1$. We claim that in this case $J_{0}^{\prime} G_{m+1}=G_{m}$ for $m \geq 4$. To prove our claim, notice that since $G_{3}=<y_{12}, G_{4}>$, then $y_{21} \equiv 1 \bmod G_{4}$ or $y_{21} \equiv y_{12} \bmod G_{4}$. Using commutator relations (cf. [2], [7]) it follows that $G_{4}=<y_{13}, G_{5}>C J_{n}^{\prime} G_{5} C G_{4}$, and therefore $J_{0}^{\prime} G_{5}=G_{4}$. Proceeding by induction, we obtain that $J_{0}^{\prime} G_{6}=G_{5}$, and we continue in this way to establish our claim that $J_{0}{ }^{\prime} G_{m+1}=G_{m}$ for $m \geq 4$. Since $G$ is nilpotent, we conclude that $G_{4}=J_{0} G_{5}=J_{0} G_{6}=\ldots=J_{0}{ }^{\prime}$, which proves our result.

We now make use of Lemma 3 to prove our main result.

Theorem 1. Let $k$ be a real quadratic number field with discriminant $d_{k}=d_{1} d_{2} d_{3}$, where the $d_{j}, j=1,2,3$, are positive prime discriminants, $C_{2}(k) \approx\left(2^{m}, 2^{n}\right), m \geq 2, n \geq 2$, and $h_{2}\left(k_{j}\right)=h_{2}(k)$ for all three unramified quadratic extensions $k_{j}$ of $k$. Then either $\left|C_{2}\left(k^{1}\right)\right|=2$ or $d\left(C_{2}\left(k^{1}\right)\right) \geq 3$. Furthermore, $d\left(C_{2}\left(k^{1}\right)\right) \geq 3$ if and only if $h_{2}\left(K_{0}\right) \geq h_{2}(k)$, where $K_{0}=k_{1} k_{2} k_{3}$. 
Proof. From Theorem 5 of [2] we see that $h_{2}\left(K_{0}\right) \neq h_{2}(k)$ (notice that $K_{0}$ is the fixed field of $J_{0}$ ), and this implies by Corollary 1 of [2] that either $\left|C_{2}\left(k^{1}\right)\right| \leq 2$ or $C_{2}\left(k^{1}\right.$ is not cyclic. Since $h_{2}\left(k_{j}\right)=h_{2}(k)$ for $i=1,2,3$, we know from Corollary 1 above that $C_{2}\left(k^{1}\right) \neq 1$. Furthermore, $h_{2}\left(K_{0}\right) \neq h_{2}(k)$ implies that $\left(G_{2}: J_{0}^{\prime}\right) \neq 4$, and therefore from Lemma 3 we know that $d\left(G_{2}\right)=d\left(C_{2}\left(\mathrm{k}^{1}\right)\right) \neq 2$. This implies that either $\left|C_{2}\left(k^{1}\right)\right|=2$ or $d\left(C_{2}\left(k^{1}\right)\right) \geq 3$, and from Corollary 1 we obtain that $d\left(C_{2}\left(k^{1}\right)\right) \geq 3$ if and only if $h_{2}\left(K_{0}\right) \geq h_{2}(k)$, which establishes our theorem.

In the case where $h_{2}\left(k_{i}\right)=h_{2}(k)$ for exactly two $k_{i}$, we have not been able to distinguish between $d\left(C_{2}\left(k^{1}\right)\right)=2$ and $d\left(C_{2}\left(k^{1}\right)\right) \geq 3$ in general, but we have obtained a preliminary result in this direction (however, see Remark 1 below), which we formulate initially with the following group theoretical lemma, where the case $h_{2}\left(k_{i}\right)=h_{2}(k)$ for exactly two $k_{i}$ is equivalent to $c=2$ (see above), and $G^{a b}$ denotes the abelianzation $G / G^{\prime}$ for any group $G$.

Lemma 4. Let $G$ be a finite metabelian 2-group of rank 2, and assume that $d\left(G_{2}\right)=2$ and $c=2$. Then $\left(G_{2}: G_{3}\right) \geq 4$ and $\left|J_{0}{ }^{a b}\right| \geq\left|G^{a b}\right|$, $\left|J_{u v}^{a b}\right| \geq\left|G^{a b}\right|$ for all $u=1,2,3$ and $v=1,2$.

Proof. We see that $\left(G_{2}: G_{3}\right) \geq 4$ from Theorem 2 of [2]. Let $J=J_{0}$ or $J_{u v}$ for $u=1,2,3$ and $v=1,2$. Since $\left(G_{2}: G_{3}\right) \geq 4$, from the formulations of $J_{0}^{\prime}$ and $J_{u v}{ }^{\prime}$ given in [2], we have $\left(G_{2}: J^{\prime}\right) \geq\left(G_{2}: G_{2}{ }^{4}\right.$ $\left.G_{3}\right)=4$ and thus $\left|J^{a b}\right| \geq\left|G^{a b}\right|$. 
Lemma 4 has the following field theoretical corollary.

Corollary 2. Let $k$ be a real quadratic number field with discriminant $d_{k}=d_{1} d_{2} d_{3}$, where the $d_{j}, j=1,2,3$, are positive prime discriminants, $C_{2}(k) \approx\left(2^{m}, 2^{n}\right), m \geq 2, n \geq 2$, and assume that $h_{2}\left(k_{i}\right)$ $=h_{2}(k)$ for exactly two $k_{i}$. Furthermore, assume that $h_{2}(K)=h_{2}(k) / 2$ for some unramified quadratic extension $K$ of $k$. Then $d\left(C_{2}\left(k^{1}\right)\right) \geq 3$.

Proof. From Theorem 6 of [2] we know that $C_{2}\left(k^{1}\right)$ is not cyclic. Therefore from the field theoretical equivalence of Lemma 4 we immediately conclude that $d\left(C_{2}\left(k^{1}\right)\right) \geq 3$.

Remark 1. From Corollary 1 above, Theorem 1 in [4], and the assumption in Corollary 2 that $h_{2}\left(k_{i}\right)=h_{2}(k)$ for exactly two $k_{i}$ we know that given the assumptions of Corollary 2, $h_{2}\left(K_{0}\right) \geq h_{2}(k)$ and that $h_{2}\left(K_{j}\right) \geq h_{2}(k)$ for the two unramified quadratic extensions $K_{j}$ of $k$ that contain $k_{j}$ with $h_{2}\left(k_{j}\right)>h_{2}(k)$. Furthermore, from a heuristic investigation (which we thank Abdelkader Zekhnini for) it appears that for fields satisfying the initial assumptions of Corollary 2 (i.e., satisfying all the assumptions of Corollary 2 with the exception that $h_{2}(K)=$ $h_{2}(k) / 2$ for some unramified quadratic extension $K$ of $\left.k\right), h_{2}\left(K_{i}\right) \geq h_{2}(k)$ for all unramified quadratic extensions $K_{i}$ of $k$. However, we have not been able to prove this expected result, and we therefore formulate the following open question.

Open Question 1. Let $k$ satisfy the initial assumptions of Corollary 2. Then does it follow that $h_{2}\left(K_{i}\right) \geq h_{2}(k)$ for all unramified quadratic extensions $K_{i}$ of $k$ ? 
Remark 2. Even if there are fields $k$ that satisfy all the assumptions of Corollary 2, i.e., if the answer to Open Question 1 is that there exists at least one real quadratic number field $k$ as above such that $h_{2}(K)=h_{2}(k) / 2$ for some unramified quadratic extension $K$ of $k$, then we still are not able to distinguish in general between $d\left(C_{2}\left(k^{1}\right)\right)=2$ and $d\left(C_{2}\left(k^{1}\right)\right) \geq 3$ (see Example 6 below).

We conclude with the following open question.

Open Question 2. Let $k$ satisfy the initial assumptions of Corollary 2 , and assume that $h_{2}\left(k_{i}\right) \geq h_{2}(k)$ for all unramified quadratic extensions $K_{i}$ of $k$. Then is it possible to distinguish between $d\left(C_{2}\left(k^{1}\right)\right)=2$ and $d\left(C_{2}\left(k^{1}\right)\right) \geq 3$ ?

\section{Examples}

Example 1. Although Theorem 1 shows that there do not exist real quadratic number fields satisfying the given conditions with $d\left(C_{2}\left(k^{1}\right)\right)=2$, there are examples that satisfy the group theoretical translated conditions of Theorem 1 with $d\left(C_{2}\left(k^{1}\right)\right)=2$. To convey this we utilize Groups 128, 129, 130 of order 64 in Hall \& Senior [9] that can be characterized as follows (cf. [13]).

$$
\begin{aligned}
& \text { Group } 128=<a, b \mid a^{4}=1, b^{4}=1, y_{11}^{2}=1,\left[y_{11}, b^{2}\right]=1,\left[y_{11}, b a\right. \\
& \left.b^{-1}\right]=1>
\end{aligned}
$$

$$
\begin{aligned}
& \text { Group } 129=<a, b \mid a^{4}=1, y_{11}^{2}=1, y_{12}=b^{4}, y_{21}=1> \\
& \text { Group } 130=<a, b \mid b^{4}=1, y_{11}^{2}=1, y_{12}=a^{4}, y_{21}=1>
\end{aligned}
$$


For all three above groups $G$, we obtain $G / G^{\prime} \approx(4,4), G^{\prime} \approx(2,2), y_{21}$ $=1, G_{3}=<y_{12}>, J_{0}{ }^{\prime}=J_{11}{ }^{\prime}=J_{12}{ }^{\prime}=1$, and ${H_{1}}^{2}={H_{2}}^{2}={H_{3}}^{2}=J_{21}{ }^{\prime}=$ $J_{22}{ }^{\prime}=J_{31}{ }^{\prime}=J_{32}{ }^{\prime}=<y_{12}>$.

In the following examples, we have made use of calculations available through pari and Keith Mathews' number theory site (www.numbertheory.org) to obtain our 2-class numbers, and we use the notation given in Lemma 2 for our unramified quadratic cyclic extensions $K_{i j}$ of $k$. For all these examples we obtain that $\left|C_{2}\left(k^{1}\right)\right|=1$ or 2 , or $d\left(C_{2}\left(k^{1}\right)\right) \geq 3$. See Example 3 in [2] for an example with $c=1$ and $d\left(C_{2}\left(k^{1}\right)\right)=2$.

Example 2. $k=Q(\sqrt{ }(2.17 .89)), C_{2}(k) \approx(4,4), h_{2}\left(k_{1}\right)=h_{2}\left(k_{2}\right)=h_{2}$ $\left(k_{3}\right)=8$; from Corollary 1 we see that $C_{2}\left(k^{1}\right)$ is trivial.

Example 3. $k=Q(\sqrt{ }(5.61 .241)), C_{2}(k) \approx(4,4), h_{2}\left(K_{0}\right)=8, h_{2}\left(k_{1}\right)=$ $h_{2}\left(k_{2}\right)=h_{2}\left(k_{3}\right)=16$ and thus $c=3$; from Corollary 1 we see that $\left|C_{2}\left(k^{1}\right)\right|=2$.

Example 4. $k=Q(\sqrt{ }(2.41 .2833)), C_{2}(k) \approx(4,8), h_{2}\left(K_{0}\right)=64, h_{2}\left(k_{1}\right)=$ $h_{2}\left(k_{2}\right)=h_{2}\left(k_{3}\right)=32$ and thus $c=3$; from Corollary 1 and Theorem 1 we see that $d\left(C_{2}\left(k^{1}\right)\right) \geq 3$.

Example 5. $k=Q(\sqrt{ }(5.401 .541)), C_{2}(k) \approx(4,8), h_{2}\left(K_{0}\right)=128 ;$ let $d_{1}=5, d_{2}=401, d_{3}=541$; then $h_{2}\left(k_{1}\right)=h_{2}\left(k_{3}\right)=32, h_{2}\left(k_{2}\right)=64$, and thus $c=2, h_{2}\left(K_{11}\right)=h_{2}\left(K_{12}\right)=h_{2}\left(K_{31}\right)=h_{2}\left(K_{32}\right)=32, h_{2}\left(K_{21}\right)=128$, $h_{2}\left(K_{22}\right)=512$; from Corollary 1 of [2] we know that $C_{2}\left(k^{1}\right)$ is not cyclic. From pari we also obtain that $C_{2}\left(K_{21}, K_{22}\right) \approx(4,8,8,8)$ and $K_{21}, K_{22}$ is an unramified degree 8 extension of $k$. We therefore are able to conclude for this example that $d\left(C_{2}\left(k^{1}\right)\right) \geq 3$. 
Example 6. $k=Q(\sqrt{ }(5.89 .1709)), C_{2}(k) \approx(4,8), h_{2}\left(K_{0}\right)=64$; let $d_{1}=5, d_{2}=89, d_{3}=1709$; then $h_{2}\left(k_{2}\right)=h_{2}\left(k_{3}\right)=32, h_{2}\left(k_{3}\right)=64$, and thus $\quad c=2, h_{2}\left(K_{21}\right)=h_{2}\left(K_{22}\right)=h_{2}\left(K_{31}\right)=h_{2}\left(K_{32}\right)=32, h_{2}\left(K_{11}\right)=64$, $h_{2}\left(K_{12}\right)=128$. From Theorem 6 in [2], we see that $C_{2}\left(k^{1}\right)$ is not cyclic, but in this example our methods do not enable us to determine if $d\left(C_{2}\left(k^{1}\right)\right)=2$ or $d\left(C_{2}\left(k^{1}\right)\right) \geq 3$.

Remark 3. We notice that in Examples 5 and 6, since $h_{2}\left(K_{0}\right) \geq h_{2}(k)$ and $h_{2}\left(K_{i j}\right) \geq h_{2}(k)$ for all $i, j$, that this is consistent with our conjecture in Remark 1 (see Open Question 1). We also note that in Example 4 above, and in Example 4 in [2] $(k=Q(\sqrt{ }(17.53 .661)))$, we have this same consistency with our conjecture in Remark 1.

\section{Acknowledgement}

We thank Chip Snyder for his continued collaboration and in parrticular for his proof of Lemma 3. We thank Abdelkader Zehkini for his class group calculations, which was useful to us for some of our above examples, and for his heuristic investigation that resulted in us making our conjecture in Remark 1 (see Open Question 1).

\section{References}

[1] E. Benjamin, Corrigendum to Some real quadratic number fields with their Hilbert 2-class field having cyclic 2-class group, Journal of Number Theory 173 (2017), 529-546; Corrigendum, JNT (2017).

DOI: https://doi.org/10.1016/j.jnt.2016.09.030

[2] E. Benjamin and C. Snyder, On the rank of the 2-class group of the Hilbert 2-class field of some quadratic fields, Quarterly Journal of Mathematics (to appear).

DOI: https://doi.org/10.1093/qmath/hay021

[3] E. Benjamin and C. Snyder, Classification of metabelian 2-groups $G$ with $G^{a b} \approx\left(2,2^{n}\right), n \geq 2$, and $\operatorname{rank} d\left(G^{\prime}\right)=2$; Applications to real quadratic number fields, Journal of Pure and Applied Algebra (to appear).

DOI: https://doi.org/10.1016/j.jpaa.2018.03.004 
[4] E. Benjamin and C. Snyder, Some real quadratic number fields whose Hilbert 2-class fields have class number congruent to 2 modulo 4, Acta Arithmetica 177 (2017), 375-392.

DOI: https://doi.org/10.4064/aa8485-9-2016

[5] E. Benjamin, F. Lemmermeyer and C. Snyder, Real quadratic fields with Abelian 2-class field tower, Journal of Number Theory 73(2) (1998), 182-194.

DOI: https://doi.org/10.1006/jnth.1998.2291

[6] N. Blackburn, On prime-power groups in which the derived group has two generators, Math. Proc. Cambridge Philos. Soc. 53(1) (1957), 19-27.

DOI: https://doi.org/10.1017/S0305004100031959

[7] N. Blackburn, On prime-power groups with two generators, Math. Proc. Cambridge Philos. Soc. 54(3) (1958), 327-337.

DOI: https://doi.org/10.1017/S0305004100033521

[8] R. Coutere and A. Derhem, Un problém de capitulation, C. R. Acad. Sci. Séerie 1314 (1992), 785-788.

[9] M. Hall and J. K. Senior, The Groups of Order $2^{n}(n \leq 6)$, Macmillan, New York, 1964.

[10] D. Gorenstein, Finite Groups, Harper \& Row, New York, 1968.

[11] B. Huppert, Endliche Gruppen I, Springer-Verlag, 1967.

[12] F. Lemmermeyer, Class Field Towers, 2010.

Retrieved from: http://www.rzuser.uni-heidelberg.de/ hb3/publ/pcft.pdf

[13] T. W. Saga and J. W. Wamsley, Minimal presentations for groups of order $2^{n}, n \leq 6$, Journal of the Australian Mathematical Society 15(4) (1973), 461-469.

DOI: https://doi.org/10.1017/S1446788700028810 